This item was submitted to Loughborough's Research Repository by the author.

Items in Figshare are protected by copyright, with all rights reserved, unless otherwise indicated.

\title{
Microstructural analysis of non-woven fabrics using scanning electron microscopy and image processing. Part 1: development and verification of the methods
}

\section{PLEASE CITE THE PUBLISHED VERSION}

\section{PUBLISHER}

(C) Professional Engineering Publishing

\section{VERSION}

VoR (Version of Record)

LICENCE

CC BY-NC-ND 4.0

\section{REPOSITORY RECORD}

Ghassemieh, E., Memis Acar, and Hendrik K. Versteeg. 2019. "Microstructural Analysis of Non-woven Fabrics Using Scanning Electron Microscopy and Image Processing. Part 1: Development and Verification of the Methods". figshare. https://hdl.handle.net/2134/4532. 
This item was submitted to Loughborough's Institutional Repository (https://dspace.lboro.ac.uk/) by the author and is made available under the following Creative Commons Licence conditions.

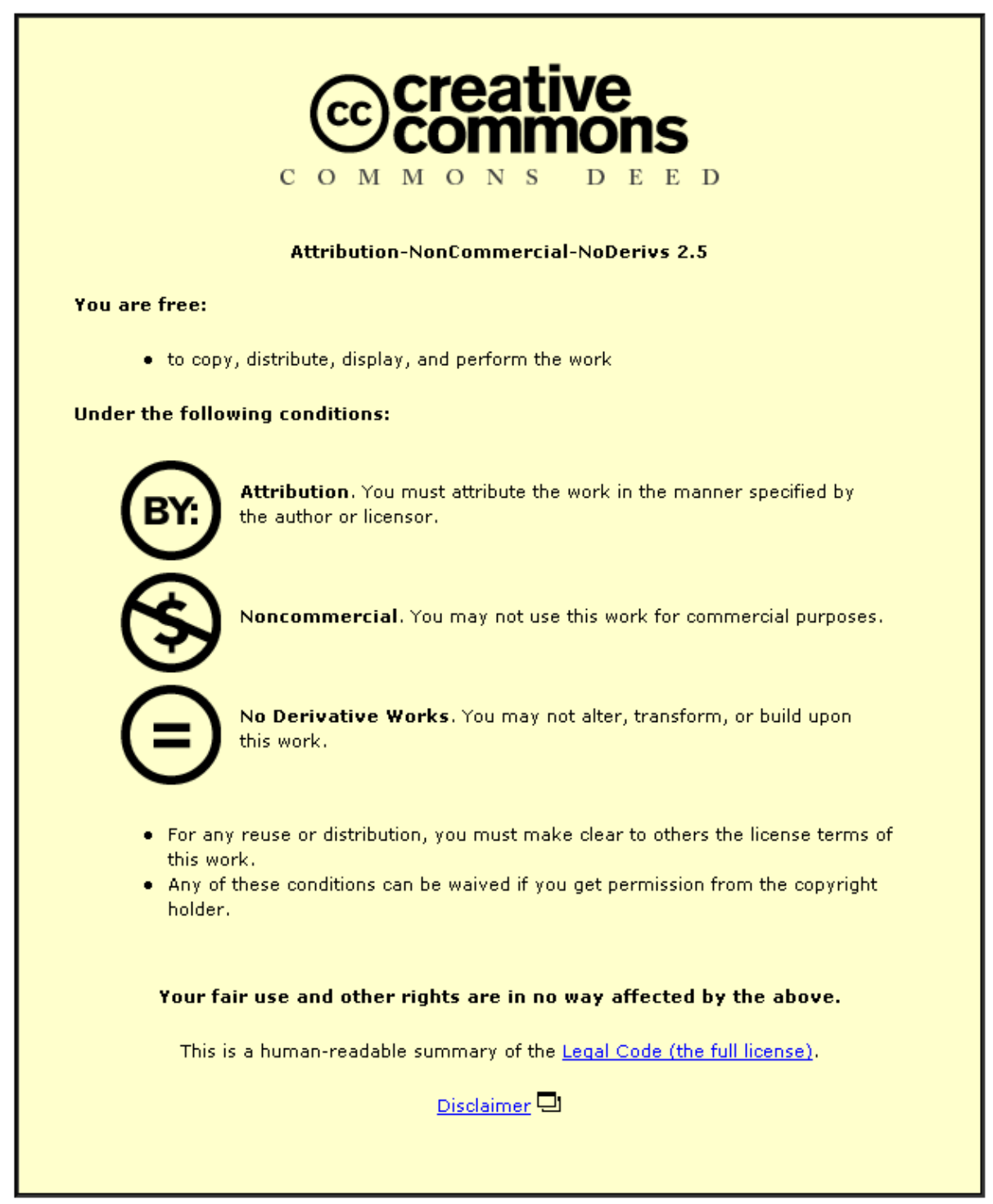

For the full text of this licence, please go to: http://creativecommons.org/licenses/by-nc-nd/2.5/ 


\title{
Microstructural analysis of non-woven fabrics using scanning electron microscopy and image processing. Part 1: development and verification of the methods
}

\author{
E Ghassemieh*, M Acar and H Versteeg \\ Mechanical and Manufacturing Engineering, Loughborough University, Loughborough, UK
}

\begin{abstract}
This paper reports image analysis methods that have been developed to study the microstructural changes of non-wovens made by the hydroentanglement process. The validity of the image processing techniques has been ascertained by applying them to test images with known properties. The parameters in preprocessing of the scanning electron microscope (SEM) images used in image processing have been tested and optimized. The fibre orientation distribution is estimated using fast Fourier transform (FFT) and Hough transform (HT) methods. The results obtained using these two methods are in good agreement. The HT method is more demanding in computational time compared with the Fourier transform (FT) method. However, the advantage of the HT method is that the actual orientation of the lines can be concluded directly from the result of the transform without the need for any further computation. The distribution of the length of the straight fibre segments of the fabrics is evaluated by the HT method. The effect of curl of the fibres on the result of this evaluation is shown.
\end{abstract}

Keywords: non-woven, fast Fourier transform, Hough transform, microstructure, scanning electron microscope

\section{INTRODUCTION}

Image processing techniques are widely used to evaluate microstructural parameters of non-woven fabrics such as fibre orientation distribution, diameter distribution, fibre crimp, fabric non-uniformity and defects. The most important microstructural aspects of non-woven fabrics relating to the fibres used in the production of the fabric are fibre diameter, length, crimp and cross-sectional shape. The geometrical parameters at micro scale relating to the fabric structure are fibre orientation distribution, curl distribution and number of fibre contact points.

Various techniques are used to estimate fibre orientation distribution in non-wovens, such as direct tracking, flowfield analysis and the Fourier transform (FT) and Hough transform (HT) methods. In direct tracking, the actual pixels of the lines are tracked [1]. The algorithm used is time consuming and therefore is mostly used for research and

The MS was received on 20 November 2001 and was accepted after revision for publication on 16 April 2002.

*Corresponding author: Wolfson School of Manufacturing and Mechanical Engineering, Loughborough University, Loughborough, Leicestershire, LE11 3TU, UK. development. In denser structures, as the number of crossover points increases, this method becomes less efficient.

Flowfield analysis is based on the assumption that edges in an image are representative of orientation fields in the image. This method is mainly used to obtain the mean orientation distribution angle, but not the orientation distribution function [2].

The FT method is widely used in many image processing and measurement operations. It transforms the grey scale intensity domain to a frequency spectrum [3]. Compression of the image, levelling of the image brightness and contrast and enhancement can more conveniently be performed on the frequency spectrum. In most cases the spatial domain needs to be recovered from the frequency spectrum. Measuring the orientation is one of the applications of the FT method. The algorithm is fast and does not need high computational power for the calculations.

The HT method is used in estimating fibre orientation distribution directly [4]. The advantage of this method compared with other indirect methods is that the actual orientation of the lines is included directly in the computation of the transform. The capability of the HT method in object recognition is used to measure the length of the straight lines in the image. However, more computational 
time and resources are needed to run the algorithm for larger-scale images with higher accuracy.

Pourdeyhimi et al. $[\mathbf{1 , 5}$ ] first tested these methods on simulated images of non-woven webs and then applied them to images of real webs. They reported a number of problems, mostly relating to image acquisition, in extending the methods to real webs. They experimented with a number of lighting systems, including fibre optics back lighting and various optical microscopes with dark-field as well as bright-field capability and various polarizing filters. The images acquired using backlighting and optical microscopes were reported to be of low quality having poor contrast. Consequently, they limited the image acquisition technique to very thin (lightweight) webs for which they could obtain images that could be successfully processed [5].

When images are digitized at high magnification, the depth of field is poor, resulting in fuzzy fibre segments and inferior image quality. To overcome this problem, in the present work a scanning electron microscope (SEM) has been used to produce high quality images. The SEM provides high depth of field even at high magnifications. This technique is not restricted to thin webs and can also be used for fabrics with very high density.

In this study, the ability of the fast Fourier transform (FFT) and HT methods to evaluate the orientation distribution of simulated and real fibres in a given image sample has been evaluated. The effect of curl on the estimated length has also been investigated. The effects of different preprocessing and image acquisition parameters on the outcome of the image processing have been studied.

Part 1 describes the development of the methods and their validation by means of test samples of known orientation distribution and fibre segment length distribution. In Part 2 the application of these methods to samples of hydroentangled fabrics is reported.

\section{DESCRIPTION OF THE METHODS}

\subsection{Estimation of the fibre orientation distribution using the fast Fourier transform}

A two-dimensional FT decomposes an image from its spatial domain of intensities into the frequency domain with appropriate magnitude and phase values. The frequency domain is a space in which each image value at image position $F$ represents the amount by which the intensity values in image $I$ vary over a specific distance related to $F$. In the frequency domain, changes in image position correspond to changes in the spatial frequency or the rate at which image intensity values are changing in the spatial domain image $I$ :

$$
F(u, v)=\int_{-\infty}^{\infty} \int_{-\infty}^{\infty} f(x, y) \mathrm{e}^{-2 \pi \mathrm{i}(x u+y v)} \mathrm{d} x \mathrm{~d} y
$$

The spatial frequency domain is interesting because:

1. It highlights periodic relationships in the spatial domain.

2. Some image processing is more efficient or indeed only practical when applied in the frequency domain.

A higher rate of change in grey scale intensity will be reflected in higher amplitudes. The frequency form of the image is also shown as an image where the grey scale intensities represent the magnitude of the various frequency components [6]. A sample of the SEM image of the non-woven fabric structure is shown in Fig. 1a. A two-dimensional discrete Fourier transform (DFT) of this image results in a magnitude spectrum (Fig. 1b). As the

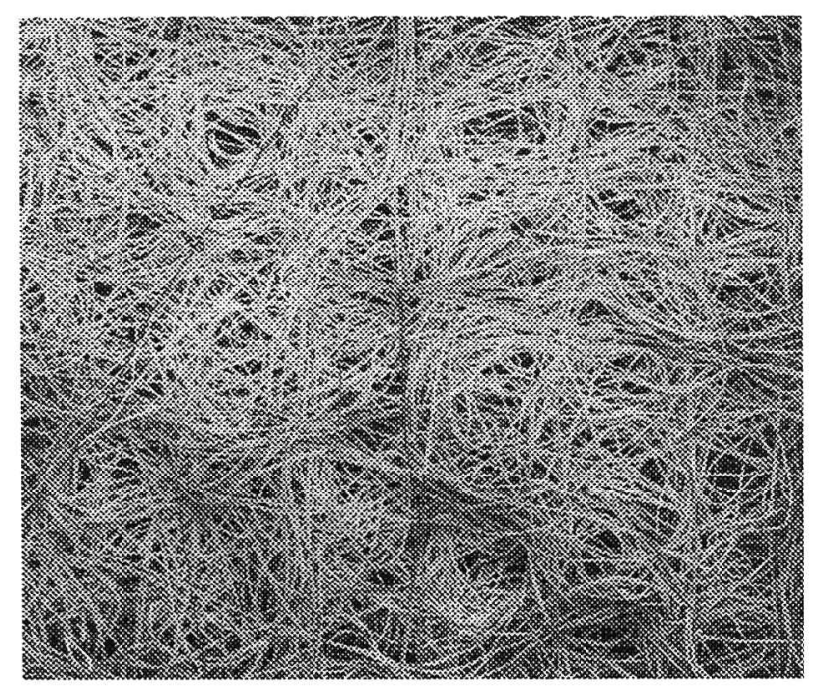

(a)

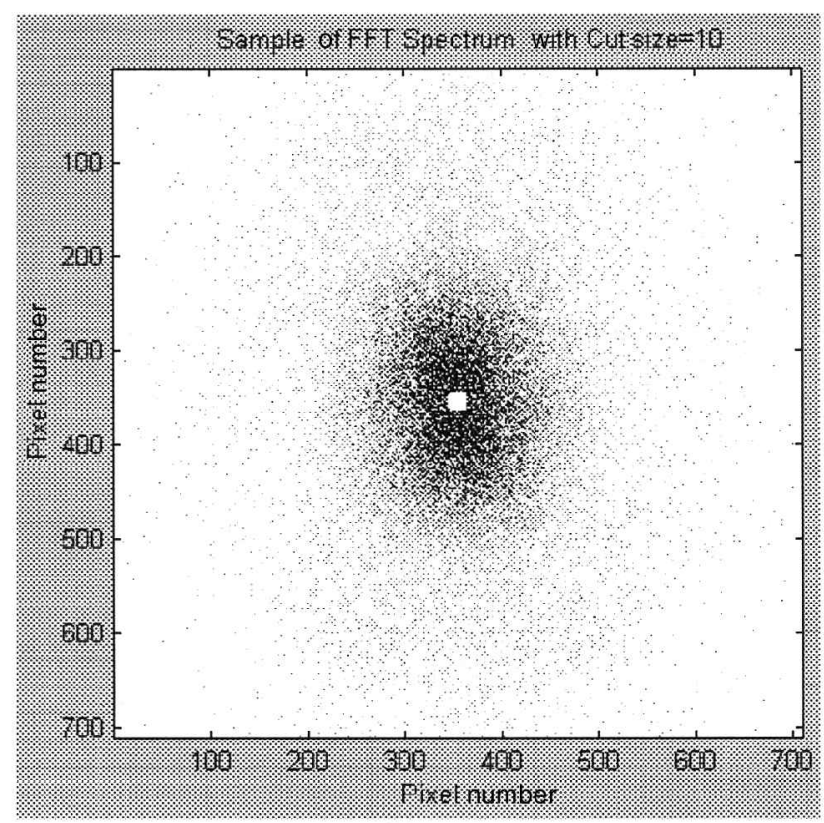

(b)

Fig. 1 (a) Sample of the SEM image of the fabric and (b) the FFT spectrum of the sample 
images are digitized, a discrete formulation of the FT is needed:

$$
F(u, v)=\frac{1}{N} \sum_{x=0}^{N-1} \sum_{y=0}^{N-1} f(x, y) \mathrm{e}^{-2 \pi \mathrm{i}(x u+y v) / N}
$$

This form can be rearranged and rewritten as follows:

$$
F(u, v)=\frac{1}{N} \sum_{x=0}^{N-1} \mathrm{e}^{-2 \pi \mathrm{i} x u / N} \sum_{y=0}^{N-1} f(x, y) \mathrm{e}^{-2 \pi \mathrm{i} y v / N}
$$

The first sum is basically just a one-dimensional DFT if $x$ is held constant. The second sum is also a one-dimensional DFT performed with the resulting values from the first set of sums. A two-dimensional DFT can be computed by performing a one-dimensional DFT for each value of $x$, i.e. for each column of $f(x, y)$, and then performing a onedimensional DFT in the opposite direction (for each row) on the resulting values. This algorithm, called the fast Fourier transform, reduces the number of transforms from $N^{2}$ to $N \log ^{2} N$ and makes the computation much faster.

The FT is useful in determining the rate at which intensity transition occurs in a given direction in the image. Thus, if the fibres are predominantly oriented in a given direction in a non-woven fabric, the spatial frequencies in that direction will be low and the spatial frequencies in the perpendicular direction will be high. This property of the FT is used to obtain information on the fibre orientation distribution in a non-woven fabric.

The transform is implemented by processing all rows one at a time, followed by all columns one at a time. The result of the transform is a two-dimensional set of values each having a magnitude and a phase. By shifting the FT results, the zero component frequency is transferred to the centre of the spectrum. Quadrants 1 and 3 are swapped with quadrants 2 and 4 respectively. The image of the magnitude spectrum is then symmetrical about the centre, and the centre represents the zero frequency. The magnitude of each frequency is indicated by the intensity of the pixel at that location (Fig. 1b). Darker areas show higher magnitudes. The zero frequency component corresponds to the average brightness of the image.

Since the centre of the spectrum contains mostly the noise in the image, the magnitude values of this section are zeroed and eliminated from the subsequent calculations. In order to find the fibre orientation distribution, an annulus of width $W$ at a radius $r$ from the centre of the image is selected. The cut-off size (the size of the central part that is eliminated) and the width affect the results and should be optimized. The width of the annulus chosen depends on the frequency ranges of interest. If an image has large values of highfrequency components, then the data change rapidly on a short distance scale. If the image has large low-frequency components, then the large-scale features of the pictures are more important. In the case of application for fibre

L04101 (C) IMechE 2002 orientation, the frequencies that are representative of the change in the grey scale across the fibre are of interest. Therefore, the cut-off size should be chosen to accommodate these frequencies.

The annulus is discretized to segments of $10^{\circ}$. In each segment, the energy or power spectrum is integrated to find the total energy of the spectrum resulting from fibres with an orientation $90^{\circ}$ offset in the range of $10^{\circ}$. Since the fibre orientation is limited to a range of $0-180^{\circ}$, the calculations are in this range. In the integration process, both the original segment and its symmetric part are taken into account.

\subsection{Estimation of the fibre orientation and length distribution using the Hough transform}

HT is one of the most effective methods for object detection in an image. Because it requires the desired features to be specified in some parametric form, the classical HT is most commonly used for the detection of regular lines such as curves, circles, ellipses, etc. Different Hough spaces are used to fit different kinds of shape, and it is necessary in most cases to know the type of line, or other geometry, that is to be fitted to the data. Here, the HT method is used in detecting straight segments of fibres in the fabric.

The most commonly used method to fit a line to data points on a graph is the least-squares method. The HT method is superior to this because it minimizes the deviations of points from the line in the direction perpendicular to the line, and it deals correctly with the case of the points not being uniform along the line. The HT projects each straight line in the image to a single point, and any part of the straight line is projected to the same point [7].

Two parameters are required to define a straight line. In Cartesian coordinates, the equation of a straight line is

$$
y=m x+b
$$

where $m$ is the slope and $b$ is the intercept. Because $m$ becomes infinitely large for lines that are nearly parallel to the $y$ axis, this representation is not usually used in the HT. Instead, the polar coordinate representation of a line is used:

$$
\rho=x \cos \theta+y \sin \theta
$$

where $\rho$ is the perpendicular distance from the origin and $\theta$ is the angle with the normal. This consists of a radius $\rho$ and angle $\theta$ that define the line as shown schematically in Fig. 2a. It is possible either to allow the angle to vary from 0 to $2 \pi$ and to keep the $\rho$ positive, or to allow $\rho$ to be either positive or negative and to restrict the angle $\theta$ to a range of 0 to $\pi$.

Collinear points $\left(x_{i}, y_{i}\right)$ with $i=1, \ldots, N$ are transformed into sinusoidal curves $\rho=x_{i} \cos \theta+y_{i} \sin \theta$ in the Hough plane, which intersects at the point $(\rho, \theta)$ (Fig. 2b). Care has to be taken in quantization of the parameter space. When the bins $(\Delta \rho, \Delta \theta)$ of the Hough space are too fine, each intersection 


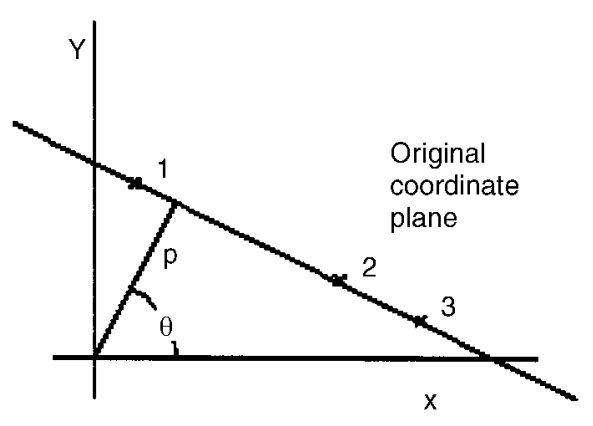

(a)

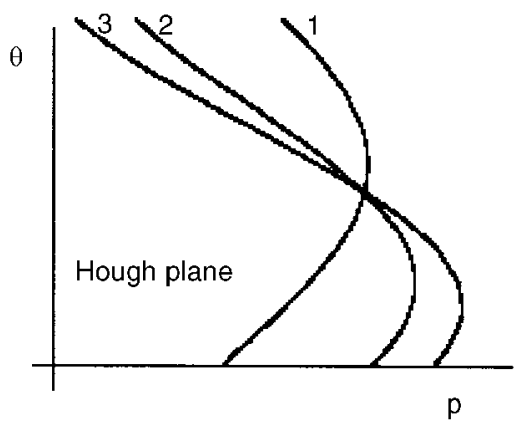

(b)

Fig. 2 (a) Sample of the line in the original coordinate system and (b) the Hough spectrum of the sample

of the sinusoidal curves can be in a different bin. When the quantization is not fine enough, on the other hand, nearly parallel lines that are close together will lie in the same bin.

For a certain range of quantized values of parameters $\rho$ and $\theta$, each $\left(x_{i}, y_{i}\right)$ is mapped into the Hough space and the points that map into the locations $\left(\rho_{\mathrm{m}}, \theta_{\mathrm{m}}\right)$ are accumulated in the two-dimensional histogram. Local maxima of the pixel intensity in the Hough space identify straight-line segments in the original image space.

When the method is applied to non-woven fabrics, firstly the pixel lines of the fibres are detected by edge detection and then all the pixels with an edge magnitude higher than the threshold are considered as fibre pixels. The binarized image of a sample of a fabric structure image is shown in Fig. 3a.

The Hough space is discretized in both the $\rho$ and $\theta$ directions. Discretization of the new space parameters changes the continuous Hough space to a rectangular structure of cells called an accumulator array. The smaller the increments in the accumulator array, the higher is the precision. However, the computational time requirement increases rapidly as the intervals in the Hough space are reduced. Therefore, the size of the intervals should be optimized to the desired accuracy and computational time.

Lines of fibres detected in the image cause a high value of the corresponding cell in the accumulator array. The cell values depend on the number of pixels or the length of the line of fibre that relates to that cell. Therefore, the line of the image can be detected by finding the maxima in the accumulator space. Through a thresholding process, values equal to or greater than a fixed percentage of the global maxima are kept and the rest are zeroed.

The values of the cells can be correlated to the length of the fibres. The number of fibres in the same range of length is counted, and a histogram of the fibre length distribution is obtained. The location of the high-value cell depends on the orientation and location of the line in the original image. The resultant HT analysis is presented in Fig. 3b. The main advantage of the HT method is that it is tolerant to gaps in feature boundary descriptions and is relatively unaffected by image noise.

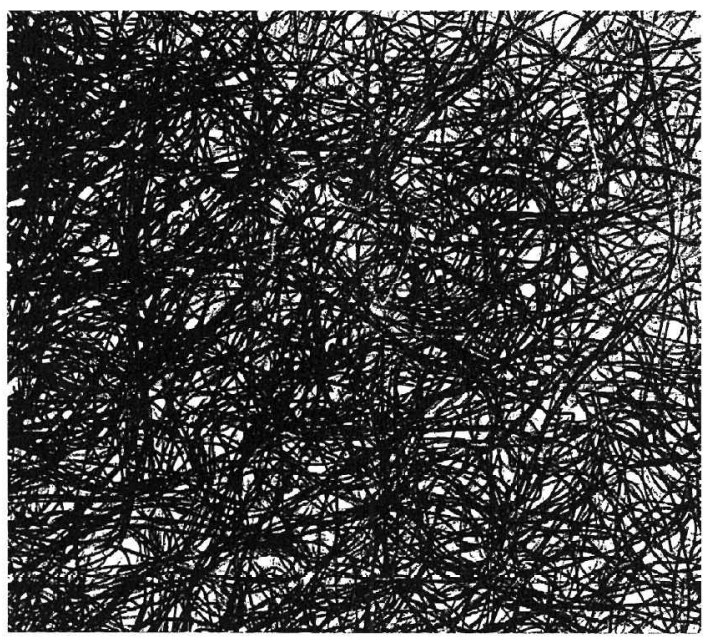

(a)

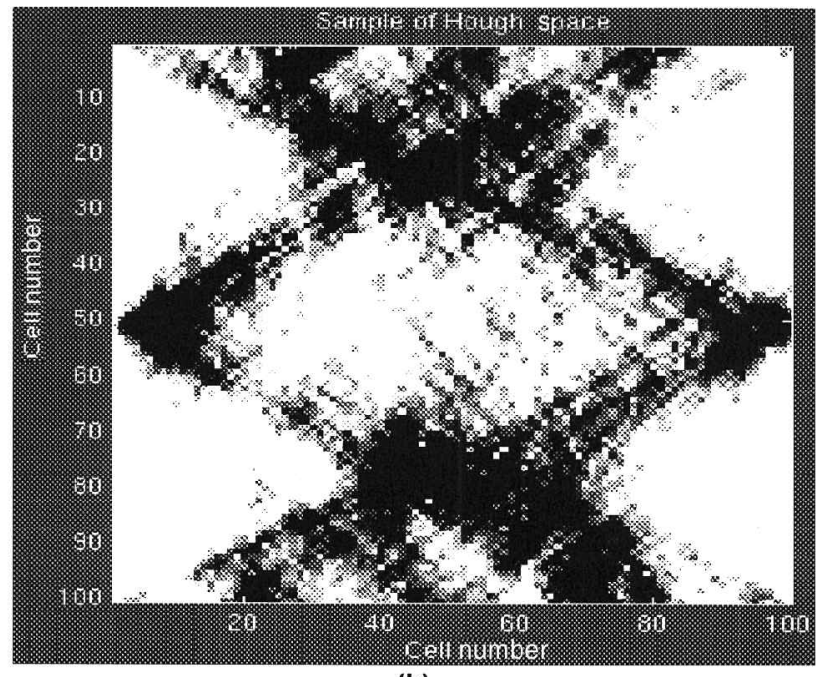

(b)

Fig. 3 (a) Sample of the binarized image of the fabric and (b) the Hough space of the sample 


\section{TEST RESULTS AND DISCUSSION}

\subsection{Test samples for validation of the methods}

In order to validate the developed techniques for their accuracy in evaluation of the fibre orientation and length distribution, several test samples consisting of lines that represent fibres in a non-woven fabric have been made. The outcomes of the image processing methods are compared with the measured microstructural parameters of the samples. Here, only one example of these samples is reported in each case. The samples chosen to be reported here have relatively randomly oriented lines. This makes the testing of the methods more rigorous compared with the samples mainly oriented in one direction, since less accuracy is required to estimate the orientation distribution in samples with mainly one dominant direction.

The orientation distribution for one of these samples (Fig. 4a) is evaluated by both the FT and HT methods. The results are compared in Fig. $4 \mathrm{~b}$ with the actual orientation of the lines manually measured for this sample. The lengths of the lines are evaluated by the HT method and the outcome is compared with the measured values in Fig. 4c.

The results of the orientation distribution from the second test sample (Fig. 5a) using the FFT and HT methods are presented in Fig. 5b and compared with the measured distribution. For this sample, the FFT results are in better agreement with the measured values than the HT results. This can be attributed to the fact that the discretization of the HT estimation is different from the FFT. Since the sample has a limited number of fibres, the orientation distribution is discontinuous and the change in the discretization intervals can have a remarkable effect on the outcome. In this sample (Fig. 5a), fibres are more randomly distributed and fibres with much more length variation can be found compared with the previous sample (Fig. 4a). This makes the estimation of the orientation distribution more complex by both methods. A finer discretization can improve the results considerably at the cost of increasing computing time.

The lengths of the lines of the sample shown in Fig. 5a are calculated by the HT method and the outcome is compared with the measured values in Fig. 5c. In the third sample (Fig. 6a) the straight lines are replaced with curved lines to evaluate the effect of curl of the lines on the method. The orientation distributions of this sample using the HT and FFT methods are shown Fig. 6b. When the fibres are curved, the orientation distributions from the FFT and HT methods do not correlate as well as those from the samples with straight lines. The length distribution of the curved line samples, estimated by the HT method, appear to be shorter in general when compared with those of the original straight lines. The deviation from mean length decreases for curved fibres, as can be seen in Fig. 6c.

The tests show that the FFT, HT and post-processing methods are able to identify correctly the orientation distribution. The HT results of the sample tests prove the validity of the method in estimating the length of the lines.

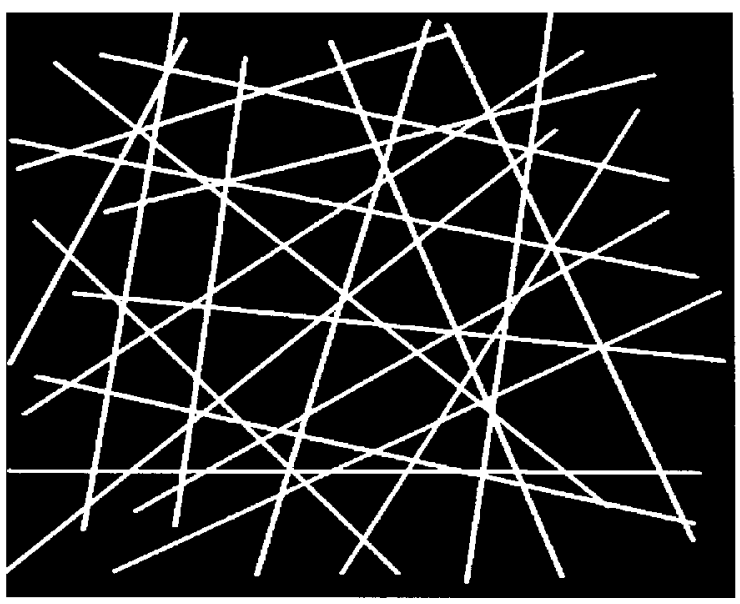

(a)

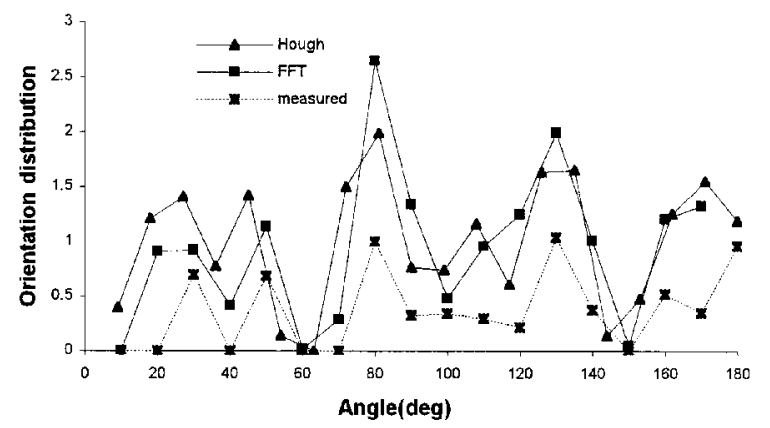

(b)

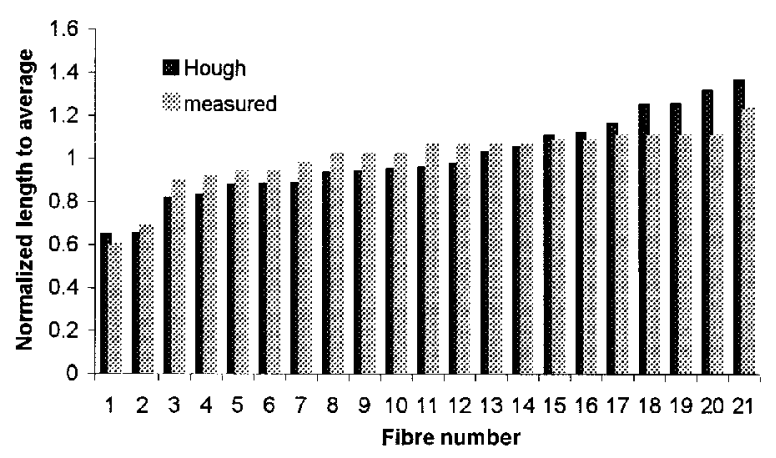

(c)

Fig. 4 (a) Test sample of lines, (b) comparison of the orientation of lines in the sample as evaluated by the FFT and HT methods with the measured values and (c) comparison of the length of the lines in the sample as evaluated by the HT method with the measured values

\subsection{Tests on image formation and preprocessing}

SEM is used to obtain images of the microstructure of the fabrics. The advantage of using the SEM in obtaining fabric images is that it has a high depth of field even at high magnifications. Using SEM, more fibres in the fabric are in focus and included in the image compared with other methods. In some cases the depth of field of the SEM can be considerably higher than that of an optical microscope [8]. 


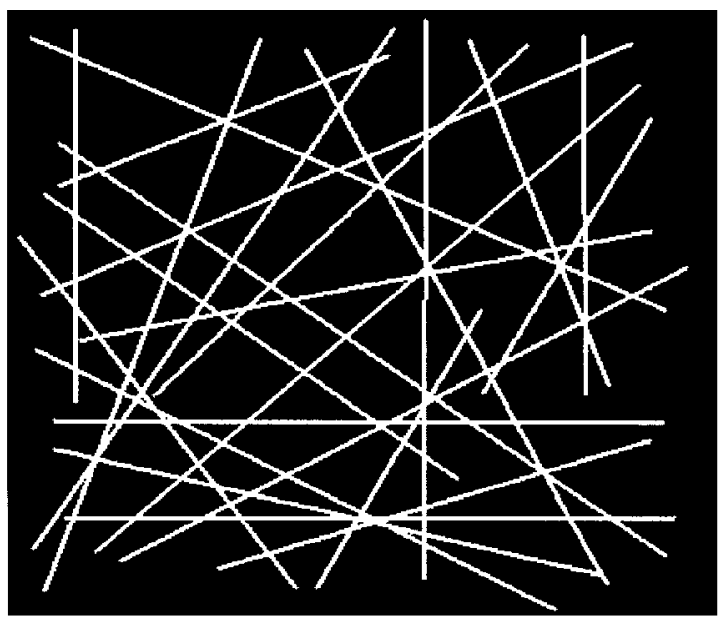

(a)

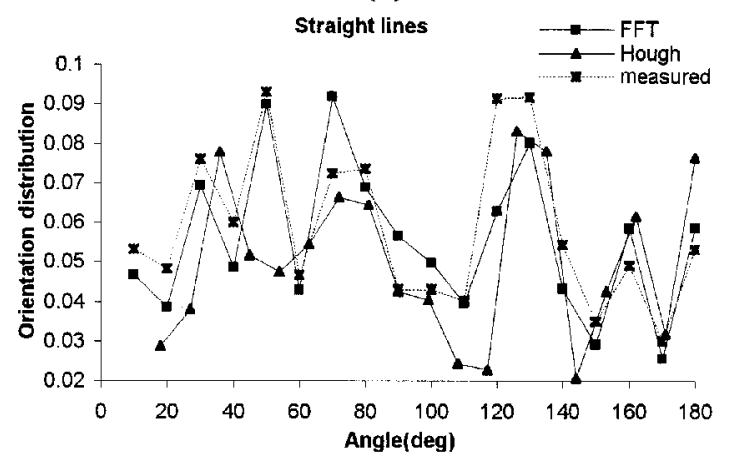

(b)

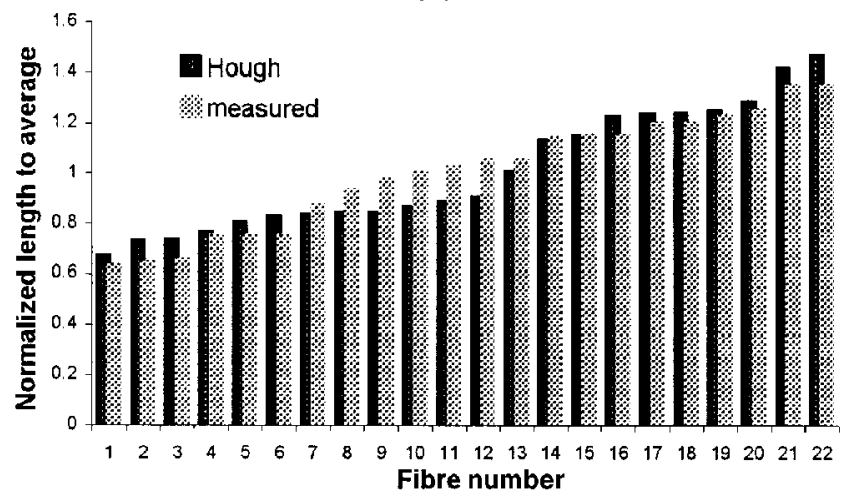

(c)

Fig. 5 (a) Test sample of lines, (b) comparison of the orientation of lines in the sample as evaluated by the FFT and HT methods with the measured values and (c) comparison of the length of lines in the sample as evaluated by the HT method with the measured values

Different processing stages have been tested to prepare the SEM images for the FFT and HT analysis.

Several parameters in obtaining SEM images and during post-processing affect the image processing and analysis. Magnification and uniformity of brightness are the most important factors at the stage of SEM image preparation. The image format and the shape of the image frame should be carefully chosen for the image analysis stage.

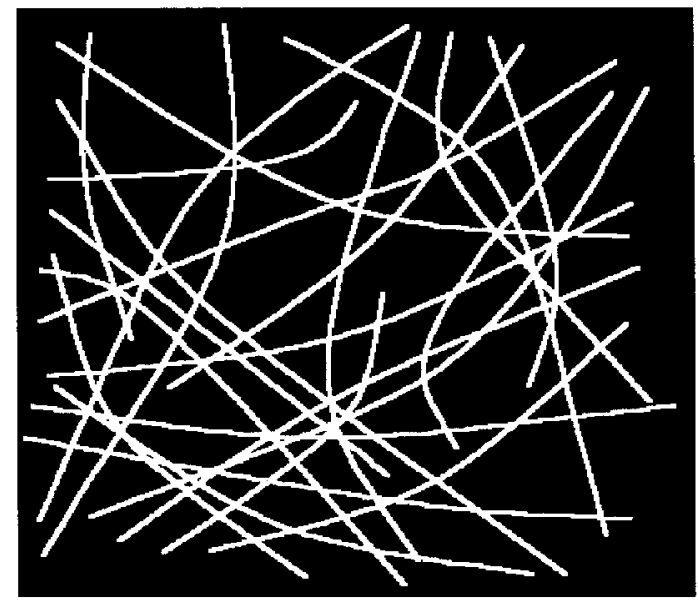

(a)

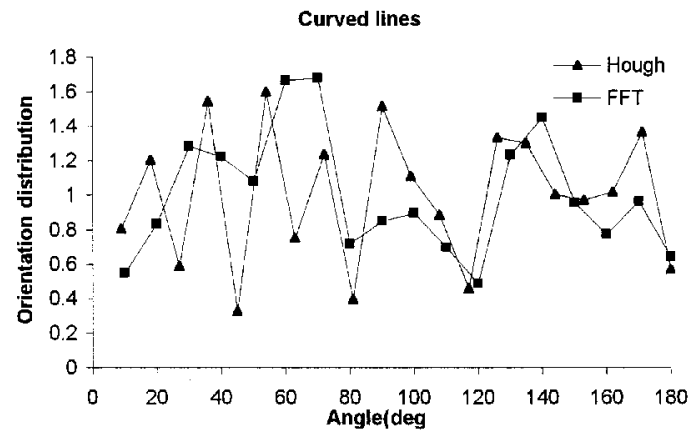

(b)

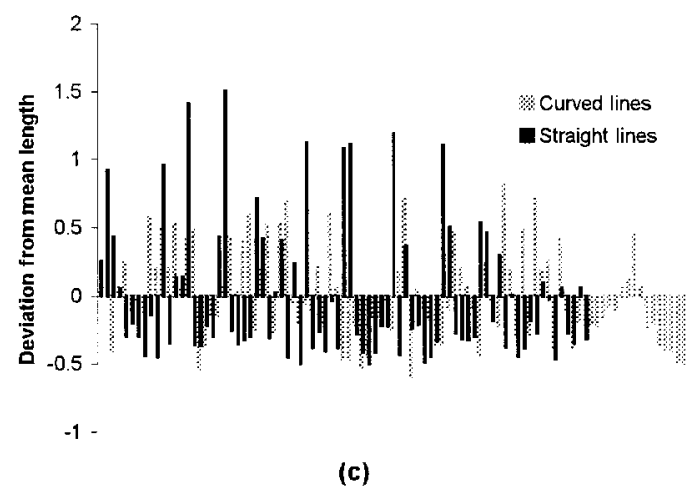

Fig. 6 (a) Test sample of curved lines, (b) comparison of the orientation of curved lines in the sample as evaluated by the FFT method with the HT results and (c) comparison of the length of curved lines in the sample with the length of the straight lines of the sample in Fig. 5a evaluated by the HT method

Each of these parameters has been studied and optimized through several tests.

\subsubsection{Sample magnification}

The magnification and area covered in an image are important factors that can affect the results. These parameters should be optimized to obtain an image that is representative 
of the whole fabric and at the same time is recognizable by the image processing methods. In the present research, magnifications of $\times 30,50$ and 100 in SEM images have been used. For each hydroentangled fabric, several images have been analysed. The samples significantly different from the average have been discarded. The results of the fibre orientation distribution by using the FFT are shown in Fig. 7 . While the $\times 30$ and 50 magnifications show similar distributions, the $\times 100$ magnification gives a completely different orientation distribution estimation. The argument is also valid for the images that are processed by the HT method. This can be attributed to the fact that, at this magnification, the image of the web used in the computations covers a smaller part of the fabric which may not be large enough to be representative of the general structure of the fabric to show all the features of the fabric microstructure. This discrepancy can be observed in the results obtained from different samples at this high magnification. It has been recommended that, for a new fabric sample, several magnifications should initially be tried to find the optimum magnitude.

\subsubsection{Image brightness}

Tests have shown that there was considerable error in the outcome of the processing whenever the brightness of the image was not uniform. Uneven brightness of the original SEM image can occur either because of overcharging parts of a sample by long exposure to electron impact or by nonuniform coating of the sample. Non-uniform brightness of an image sample can result in unequal contributions of different parts of the image to the processing outcome. SEM images of a fabric with non-uniform and uniform brightness levels are shown in Figs 8a and b respectively. The results of the fibre orientation distribution are presented in Fig. 8c, which verify that unequal brightness levels affect the results of the analysis significantly. It is therefore essential to ensure that the SEM images have uniform brightness. In the case of the HT method, a binarized

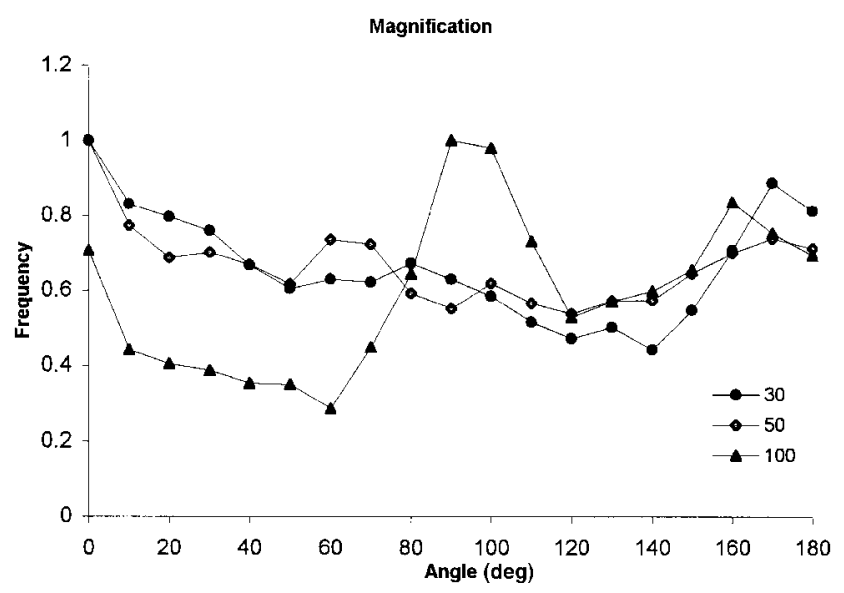

Fig. 7 Effect of magnification on the result of the fibre orientation distribution

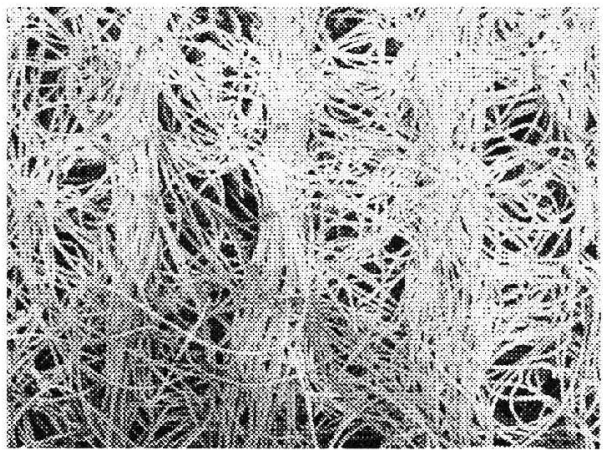

(a) Non-uniform brightness

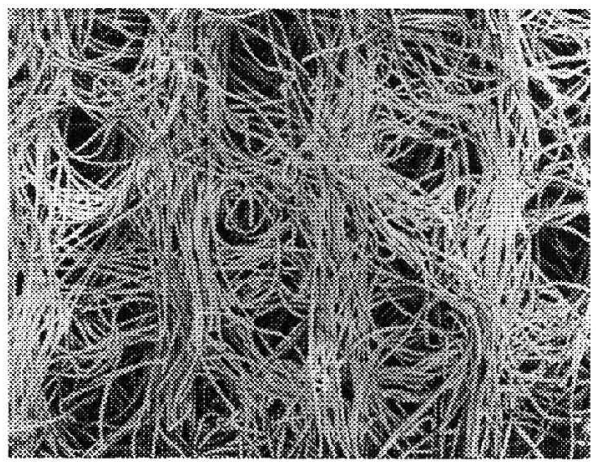

(b) Uniform brightness

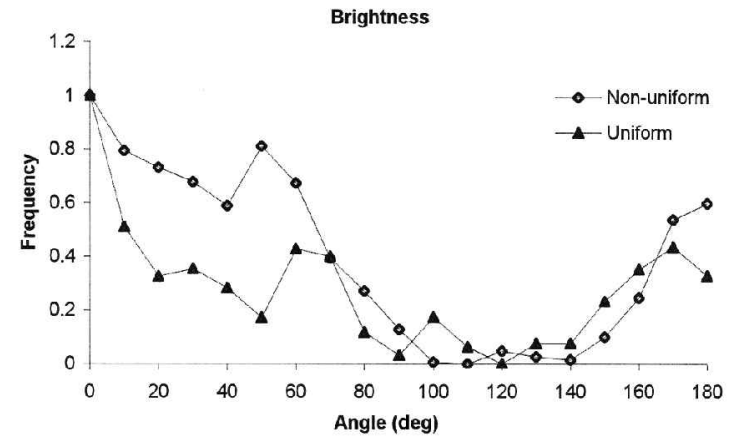

(c)

Fig. 8 (a) Sample of the SEM image with non-uniform brightness, (b) sample of the SEM image with uniform brightness and (c) the effect of non-uniform brightness on the result of the fibre orientation distribution

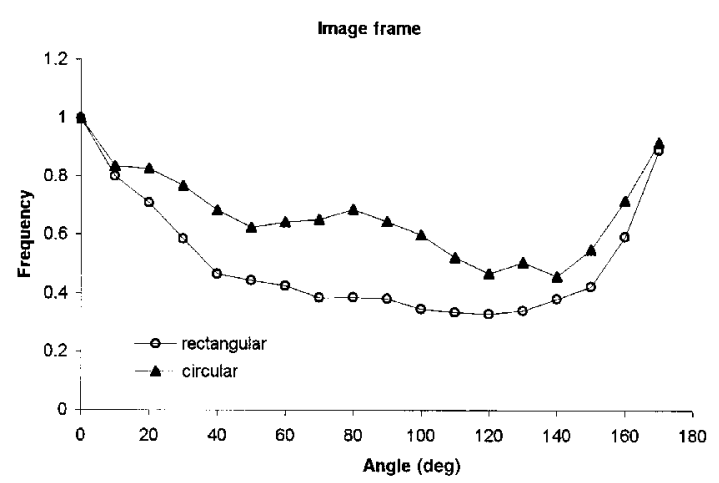

Fig. 9 Effect of the shape of the image frame on the result of the orientation distribution

Proc Instn Mech Engrs Vol 216 Part L: J Materials: Design and Applications 


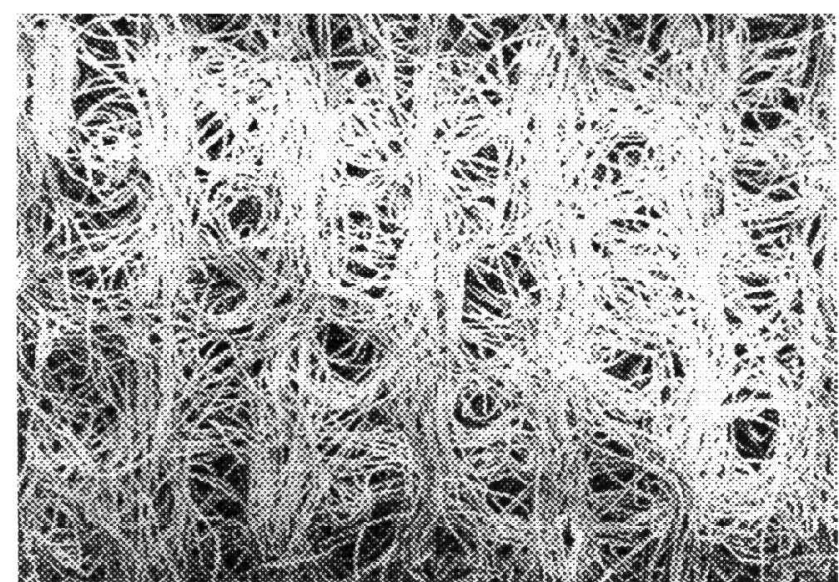

(a) Unprocessed

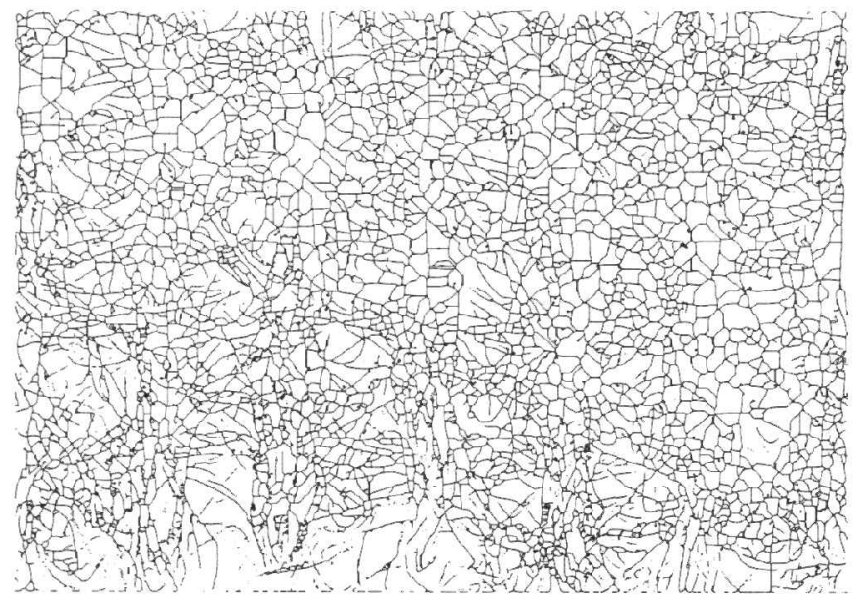

(b) Skeletonized

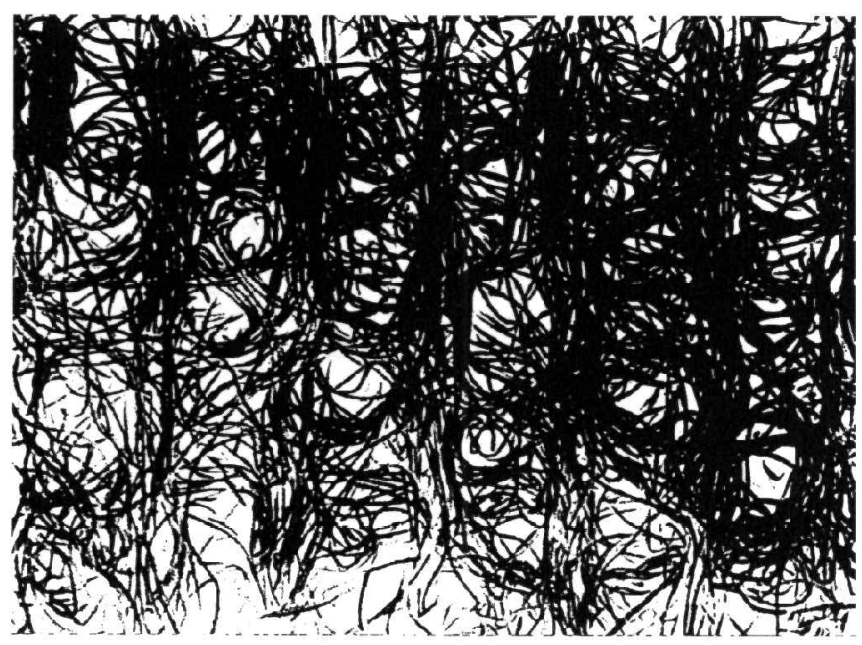

(c) Binarized

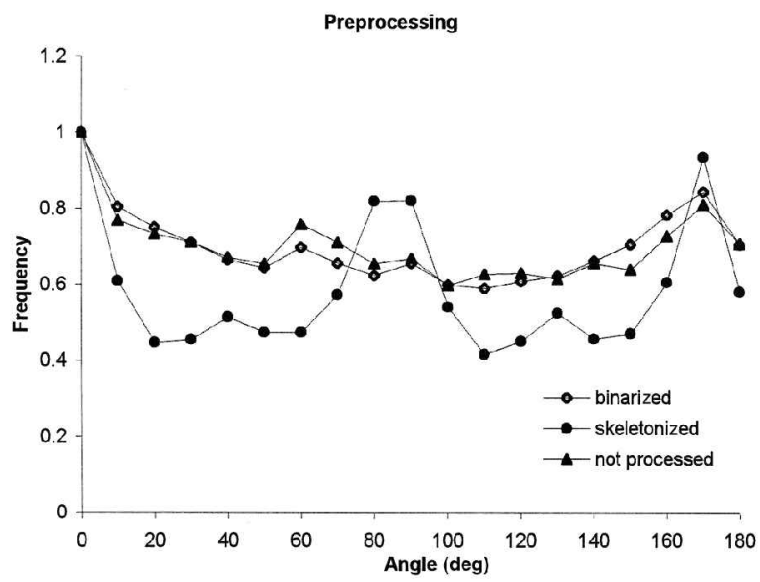

(d)

Fig. 10 (a) Sample of the unprocessed image, (b) sample of the skeletonized image, (c) sample of the binarized image and (d) the effect of preprocessing on the result of the fibre orientation distribution

image needs to be used. There is less sensitivity to the brightness level. However, major differences in the brightness in different parts of the image can result in losing part of the fibres in the binarizing process and losing the accuracy of the HT method.

\subsubsection{Shape of the image frame}

The shape of the frame of the image affects the result of the FFT image processing. Images with rectangular and circular frames have been used and the results are shown in Fig. 9. The rectangular frame clearly shows a lower percentage of fibre orientation parallel to the short edge of the image frame which is the cross-direction of the sample $\left(90^{\circ}\right)$. In a circular image frame, fibres of all orientations would have equal chance to contribute to the processing. Therefore, a circular frame is chosen as a more suitable shape for estimation of the fibre orientation distribution. However, in the HT analysis the choice of the image frame was restricted to a square shape because of the limitations in the algorithm used. This limitation is applied to increase the efficiency and speed of computation. Since the HT method is highly demanding on computing time, reducing this time is of importance in choosing the options and inputs of the algorithm.

\subsubsection{Image format}

Another important factor in image analysis is the processing of the initial image to be able to extract the necessary information by various methods. Different processing methods such as thresholding, binarizing and skeletonizing have been investigated. The fibre orientation distributions of an original unprocessed SEM image (Fig. 10a), a binarized image (Fig. 10b) and a skeletonized image (Fig. 10c) have all been estimated by the FFT method, and the results are presented in Fig. 10d. The results of the original SEM and binarized images are very close. This is especially the case when the SEM image has even brightness. The skeletonized image shows a different orientation distribution. This can be attributed to the segmentation that occurs in skeletonizing. 
Optimizing edge detection and thresholding is of primary importance in providing a skeletonized image. In the case of straight fibres, the results of the skeletonized, binarized and original images are very similar. The HT algorithm requires a binarized image.

\section{CONCLUSIONS}

Image processing techniques in conjunction with SEM images have been developed to study the microstructural changes in non-woven fabrics. The techniques have been validated by applying the methods to simulated images of non-woven fabrics with known properties. Reasonable agreement has been found between the measured and image processing estimations of orientation and length distributions. The orientation distributions evaluated by the HT and FFT methods are very close.

The key parameters such as image magnification, brightness, format and frame shape at the stage of SEM image preparation and preprocessing that affect the image analysis have been discussed. A symmetric frame shape (circular or square, for example) and uniform brightness are recommended. The magnification should be optimized before HT or FT analyses of any given fabric sample. The main criterion for the optimum magnification is that the image should be representative of the microstructural features of the fabric. Even brightness of the image is more important in FT analysis. In the HT algorithm, since a binarized image is used and the method is generally more tolerant of defects, uneven brightness has less effect on the outcome of the processing.

If the image consists mainly of straight fibres with even brightness, original SEM, binarized or skeletonized images make little difference to the FT results. However, curved fibres and uneven brightness lead to binarized and skeletonized images that are significantly different from the original
SEM image. Therefore, Fourier analysis of binarized and skeletonized images in these cases can give very different outcomes in the orientation distribution estimations. The HT algorithm requires binarized images. As the algorithm looks for straight segments of the fibres, the HT results for skeletonized and binarized images are very similar.

Since the SEM with a high depth of field has been used to obtain the images of the fabric microstructure, the image analysis methods developed can be applied to fibrous assemblies of high thickness (weight). The image analysis methods should also be applicable to the images of fibres obtained by other microscopic techniques.

\section{REFERENCES}

1 Pourdeyhimi, B. and Ramanathan, R. Measuring fibre orientation in non-wovens. Part II: direct tracking. Textile Res. J., 1996, 66(12), 747-753.

2 Pourdeyhimi, B. and Ramanathan, R. Measuring fibre orientation in non-wovens. Part IV: flow-field analysis. Textile Res. J., 1997, 67(3), 181-187.

3 Pourdeyhimi, B. and Ramanathan, R. Measuring fibre orientation in non-wovens. Part III: Fourier transform. Textile Res. J., 1997, 67(2),143-151.

$4 \mathrm{Xu}, \mathrm{B}$. and Yu, L. Determining fibre orientation distribution in non-wovens with Hough transform techniques. Textile Res. J., 1997, 67(8), 563-571.

5 Pourdeyhimi, B., Dent, R., Jerbi, A., Tanaka, S. and Deshpande, A. Measuring fibre orientation in non-wovens. Part V: real webs. Textile Res. J., 1999, 69(3), 185-192.

6 Sonka, M., Hlavac, V. and Boyle, R. Image Processing, Analysis, and Machine Vision, 1999 (PWS Publishing, Pacific Grove, California; London).

7 Russ, J. C. The Image-processing Handbook, 2nd edition, 1995 (CRC Press, Boca Raton, Florida; London).

8 Hall, C. E. Introduction to Electron Microscopy, 1996 (McGraw Hill). 